Application Forum

\title{
Map the Brain with CLARITY
}

\author{
A Novel Optical Clearing Method allows Deep Imaging of Whole Tissues \\ Isabelle Köster, PhD, Leica Microsystems
}

\section{Overview}

Image a whole brain without sectioning? Investigate neuronal circuits without reconstruction? Perform molecular phenotyping without destroying subcellular structures? Understanding the brain with molecular resolution and global scope has always been challenging.

The novel CLARITY method, developed by the Deisseroth laboratory at Stanford University, USA, pushes the barrier of deep tissue imaging a big step ahead. By making mouse brains optically transparent, while preserving the native molecular structure, it is now easily possible to investigate the subcellular structure, neuronal networks and even biomolecular complexes of a whole intact organ.

\section{Brain Imaging Methods to Date}

To date, mapping of the brain is possible only in limited ways. Due to light scattering by the tissue, conventional deep tissue imaging by multiphoton microscopy is limited to around $1 \mathrm{~mm}$ tissue depth. To investigate brain structure and cell networks located in the center, the sample has to be sectioned and investigated in small volumes. Single neuron projections need to be followed across several sliced samples. This is elaborate. New tools are constantly being developed: automated sectioning methods reduce tedious work and minimize tissue damage, and novel software facilitates analysis and reconstruction. However, reconstruction may not always be possible or sufficient for detailed analysis of neuronal circuits. New optical clearing methods using organic solvents reduce light scattering and image deeper into the tissue. But these methods leave the lipid bilayers intact, so that they still function as diffusion barrier. Penetration of light and macromolecules for whole mount staining methods is still limited.

\section{How to Achieve Brain Clarity}

CLARITY (an acronym for Clear Lipid-exchanged Acrylamide-hybridized Rigid Imaging / Immunostaining / in situ-hybridization-compatible Tissue hYdrogel) is as simple as it is brilliant. The intact tissue is transformed into a hydrogel-tissue hybrid. Similar to petrification or fossilization, the physical structure of the tissue remains intact and is prevented from disintegrating. Light-scattering lipids are removed, while proteins and nucleic acids are preserved, because they are covalently linked to the hydrogel mesh.

In a first step, the tissue is infused with acrylamide/bisacrylamide, formaldehyde and a thermosensitive starter at $4{ }^{\circ} \mathrm{C}$. Formaldehyde crosslinks the tissue and covalently links the hydrogel monomers to proteins and nucleic acids. Lipids and biomolecules lacking the functional groups are not bound and thus, can be removed. Only $8 \%$ of proteins are lost by this method (compared to $25-40 \%$ with conventional fixing and solubilization). Polymerization is triggered by elevating the temperature to $37^{\circ} \mathrm{C}$. The tissue is now a hydrogel-hybrid. The mesh supports tissue structure and its nanopores allow macromolecules to enter.

Lipid bilayers are removed by electrophoretic tissue clearing (ETC). In a custom-made electrophoresis chamber, the sample is placed in an ionic detergent (4\% SDS, sodium dodecyl sulfate) and an electric field is applied. The highly charged ionic SDS-micelles remove lipids actively. ETC has two major advantages above standard organic solubilization:

1. It does not quench fluorescence like many organic solvents.

2. Removal of lipids is fast. Passive diffusion of detergents would take up to months to completely remove the fat. transparent and is ready for imaging.

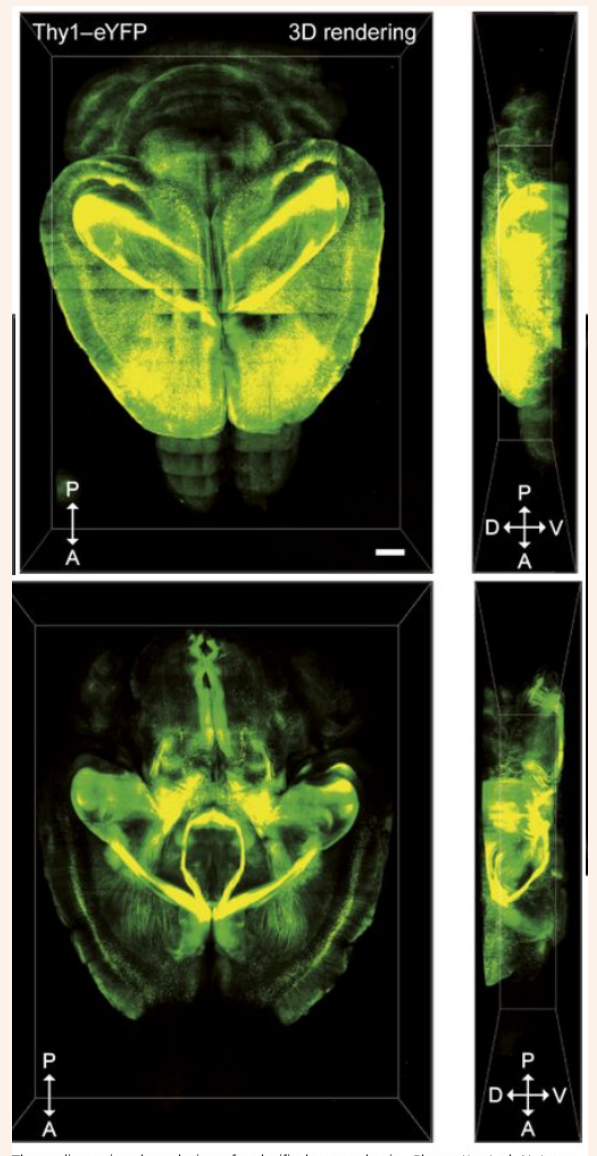

Three-dimensional rendering of a clarified mouse brain. Chung K, et al. Nature 497 (7449): 332-7 (2013) 
: Step 1:
Hydrogel Monomer Infusion $4{ }^{\circ} \mathrm{C}$

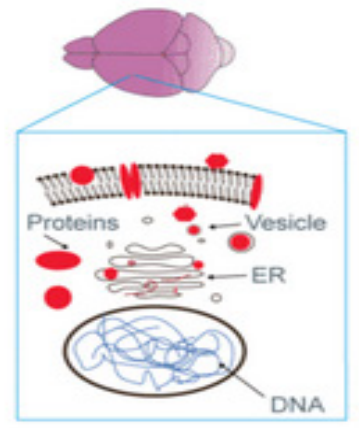

+ Formaldehyde + Hydrogel Monomer $\mathrm{CH}_{2} \mathrm{O} \quad \mathrm{C}_{2} \mathrm{H}_{5} \mathrm{~N}$
Step 2:

Hydrogel-Tissue Hybridization $37^{\circ} \mathrm{C}$
Step 3:

Electrophoretic Tissue Clearing

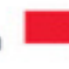

\section{.}
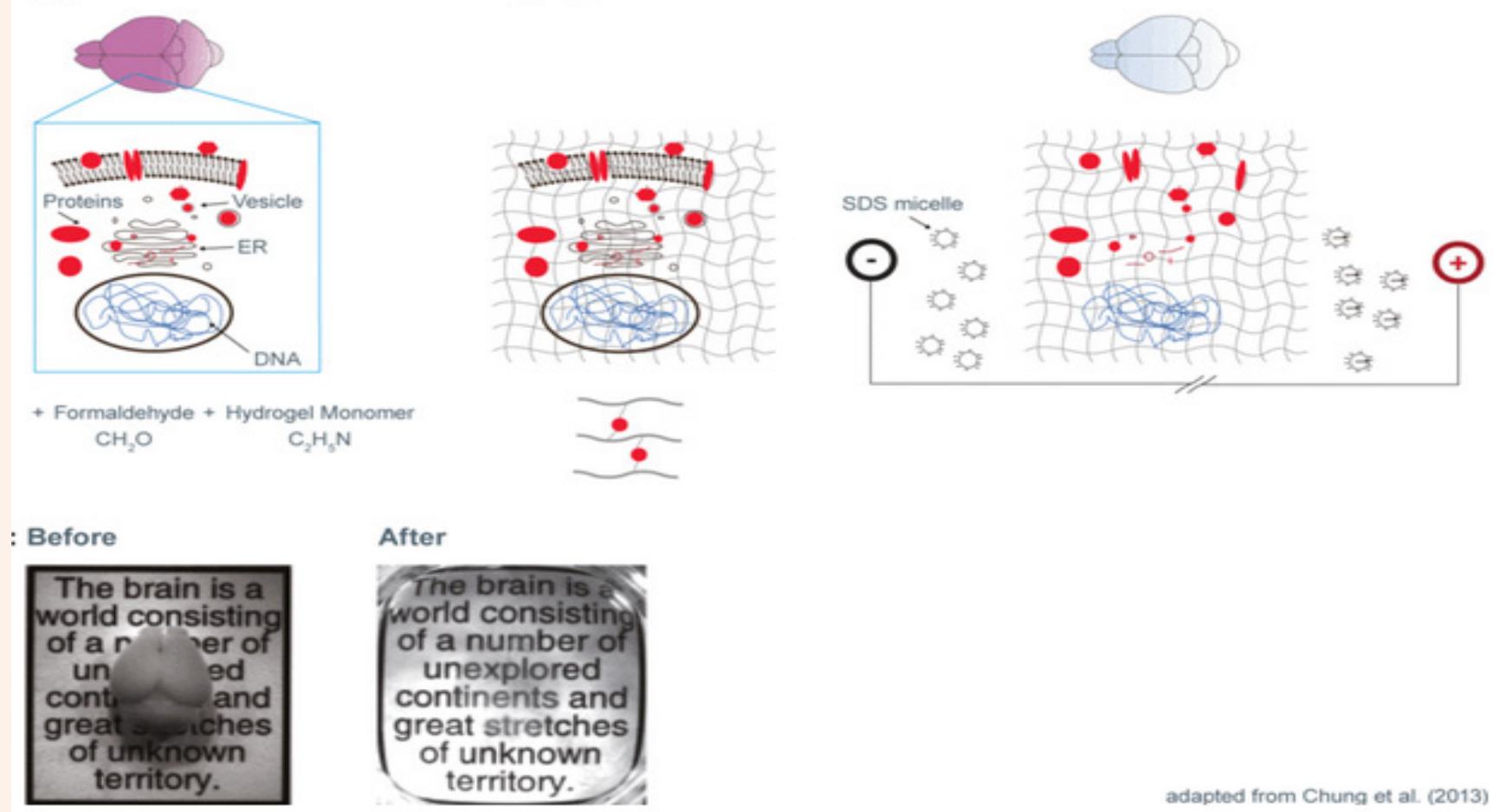

adapted from Chung et al. (2013)

Description of CLARITY method: Step 1: tissue is infused with acrylamide/bisacrylamide, formaldehyde and a thermo-sensitive starter at $4{ }^{\circ} \mathrm{C}$. Step 2 : Formaldehyde crosslinks the tissue and covalently links the hydrogel monomers to proteins and nucleic acids. Lipid bilayers are removed by electrophoretic tissue clearing (ETC).

\section{Imaging Whole Brains}

All classical laser-scanning microscopy techniques are suitable for imaging CLARITY-treated tissue, as every excitation and emission wavelength can penetrate deep into the tissue.

Imaging depth is mainly limited by the working distance of the objective. An objective of $3.6 \mathrm{~mm}$ requires two brain scans: first the dorsal half and then the ventral side. Long-distance objectives will enable to image a whole organ in one go. At the annual meeting of the Society for Neuroscience 2013, Leica Microsystems presented the latest objective development: a special CLARITY objective with motorized correction collar designed for whole organ imaging at maximal imaging depth and with highest resolution that will become available in 2014.

\section{A World of New Possibilities}

What makes CLARITY so exciting is the fact, that you can investigate whatever you want in one single brain. Protein complexes, gene expression profiling, neuronal circuits - you don't have to decide what you want to see. Look at all of it, one after the other.

The nanoporous hydrogel mesh is permeable for macromolecules and allows rapid diffusion of probes. Antibody staining is easy, even in multiple rounds. The stable framework of the hybrid allows antibodies to be washed out with SDS. In contrast to conventional elution methods using organic solvents, fluorescence of subsequent staining is not quenched. Even after 3 consecutive rounds of staining and de-staining there is no loss of image quality.

It is possible to follow axonal projections through the brain over long distances. Showing the colocalization of paired pre- and post-synaptic proteins even allows the investigation of single synapses. And after using classical fluorescent microscopy, the tissue is still appropriate for electron microscopy to observe cellular structures more closely.

The method is not limited to fresh brain tissue. It was also successfully used with long-term fixed human autopsy brain tissue or zebra fish embryos, opening new possibilities to study neurological disorders or embryonic development.

CLARITY gives both structural and molecular information and adds to the understanding of large-scale intact biological systems. In short, CLARITY revolutionizes basic research.

For Full Article visit: http://www.leica-microsystems.com/science-lab/map-the-brain-with-clarity/ 